\title{
La Formation d'une technocratie. L'École polytechnique et ses élèves de la Révolution au Second Empire
}

Jean-Luc Chappey

\section{(2) OpenEdition Journals \\ Édition électronique \\ URL : https://journals.openedition.org/ahrf/1564 \\ DOI : 10.4000/ahrf.1564 \\ ISSN : 1952-403X \\ Éditeur : \\ Armand Colin, Société des études robespierristes}

\section{Édition imprimée}

Date de publication : 1 septembre 2004

Pagination : 223-227

ISSN : 0003-4436

\section{Référence électronique}

Jean-Luc Chappey, « La Formation d'une technocratie. L'École polytechnique et ses élèves de la Révolution au Second Empire », Annales historiques de la Révolution française [En ligne], 337 | juilletseptembre 2004, mis en ligne le 15 février 2006, consulté le 22 avril 2022. URL : http:// journals.openedition.org/ahrf/1564; DOI : https://doi.org/10.4000/ahrf.1564

Ce document a été généré automatiquement le 22 avril 2022.

Tous droits réservés 


\title{
La Formation d'une technocratie. L'École polytechnique et ses élèves de la Révolution au Second Empire
}

\author{
Jean-Luc Chappey
}

\section{RÉFÉRENCE}

Bruno Belhoste, La Formation d'une technocratie. L'École polytechnique et ses élèves de la Révolution au Second Empire, Paris, Belin, 2003, 507 p., ISBN 2-7011-3523-0.

1 Prenant résolument le parti d'une histoire sociale de l'École polytechnique, Bruno Belhoste nous livre ici une étude particulièrement stimulante sur cette prestigieuse institution. S'attachant à prendre le contre-pied d'une historiographie trop souvent enfermée dans les pièges des représentations, l'auteur a non seulement pour objet d'étudier les transformations de l'institution, de son personnel et des modalités de construction et de diffusion des savoirs dont elle est le théâtre, mais aussi de s'interroger sur le rôle essentiel qu'a joué cette École dans la constitution progressive de la bureaucratie technique et de la culture technocratique durant le XIXe siècle. Par là, il s'agit de comprendre les fondements de l'autorité et du pouvoir conféré au " petit groupe » des Polytechniciens (environ 10000 élèves entre 1794 et 1871) à l'intérieur du monde étroit des élites sociales. Comme l'indique le titre, il s'agit moins de proposer une prosopographie des Polytechniciens que de réfléchir à la construction de la «technocratie », considérée comme un « univers social » avec son discours, ses codes et ses pratiques dont l'École polytechnique permet progressivement l'autonomisation. S'appuyant sur les travaux de P. Bourdieu, l'auteur s'attache donc à analyser les conditions d'émergence et de formalisation d'un «champ » technocratique qui impose progressivement sa domination durant le XIXe siècle. L'étude de la fonction instituante de l'École Polytechnique, objet de la première partie de l'ouvrage, permet de replacer l'École comme le centre d'une organisation, voire d'une « machinerie » institutionnelle au sein de laquelle se constitue un système de normes et de règles qui tracent les 
contours de la "culture technocratique ». Entre les écoles préparatoires et les diverses administrations militaires ou civiles qui se rattachent au "service public » (Poudres et Salpêtres, Marine, mais aussi des domaines civils comme les Chemins de fer ou les Télégraphes), l'École est située au cœur d'un dispositif auquel elle confère sa cohérence.

2 À travers une étude particulièrement précise du fonctionnement de la nébuleuse institutionnelle constituée autour de l'École, B. Belhoste parvient à mettre en lumière les contours du système à partir duquel se fabriquent les futurs technocrates et s'élaborent les principes cognitifs de l'action technocratique, à la croisée des domaines scientifiques, techniques et bureaucratiques. L'auteur souligne ainsi que loin d'être un simple lieu d'enseignement, l'École Polytechnique est un espace de recrutement et de formation où se construit un type social, une culture du service de l'État avec ses codes, ses pratiques et ses modes de distinction. Ce système est tout entier tourné vers une fonction : un « dispositif de triage asservi aux besoins des services publics » qui fait de l'École une véritable « institution d'État ». À partir de cette approche, c'est le processus de construction et de légitimation d'une nouvelle élite sociale imposant son pouvoir au cœur du système administratif qui est ainsi dévoilé. Créée à l'origine par le gouvernement montagnard pour former des ingénieurs, forme intermédiaire de « l'artiste » et du « savant » de l'Ancien Régime, l'École se transforme rapidement, dès l'époque directoriale (la promotion de Laplace au détriment de Monge constitue sur ce point un infléchissement important), en un lieu de formation de l'élite bureaucratique, une transformation qui rompt avec l'idéal « démocratique » des fondateurs en dépit de la référence constante à la «méritocratie» dont l'auteur offre une analyse particulièrement éclairante des enjeux. L'analyse des différentes modalités qui structurent le parcours du Polytechnicien (rites de passage, concours, contraintes collectives...) fait émerger les conditions à partir desquelles se construit l'identité du groupe, "l'esprit de corps", de cette élite qui, se distinguant fortement des autres groupes d'étudiants (juristes ou médecins), tend à constituer un véritable «État dans l'État ». La seconde partie de l'ouvrage est consacrée à l'étude des fondements de la compétence et de "l'action technocratique " à partir desquels se construisent la légitimation et la distinction sociale des Polytechniciens. Comme le souligne l'auteur, la science, et en particulier les mathématiques, tiennent une place essentielle dans la construction de la culture technocratique, une place qui ne se démentira pas pendant tout le XIXe siècle en dépit des réformes pédagogiques successives. De manière particulièrement convaincante, $B$. Belhoste montre que cette place privilégiée accordée aux mathématiques dans le cursus scolaire des Polytechniciens tient moins à un rôle strictement pédagogique qu'à des impératifs de production sociale: en clair, les mathématiques agissent comme l'outil essentiel de production et de distinction de l'élite technocratique; elles constituent ainsi la ressource première du capital scientifique, mais aussi symbolique, à partir duquel les Polytechniciens peuvent revendiquer un pouvoir, une autorité sociale et politique. À partir de l'analyse des relations établies avec l'Académie des sciences, de l'étude des carrières et des travaux des professeurs de l'École, l'auteur montre que, pendant toute la première moitié du XIXe siècle, Polytechnique, "école de savants", joue le rôle fondamental dans la construction du savoir mathématique et s'impose, au sein de la géographie parisienne des lieux de savoirs, comme un véritable « laboratoire " scientifique, à tel point que la fraction du monde savant la plus orientée vers le pôle disciplinaire des mathématiques tend à se confondre avec l'élite technocratique. En adossant la science mathématique au service de l'État, l'École joue ainsi un rôle essentiel dans la formalisation et la 
valorisation de cette discipline au sein de l'organisation des savoirs et impose la figure du professeur de mathématiques comme une figure dominante de l'espace scientifique. Autant qu'un outil de savoir, la science mathématique s'impose comme le fondement de la compétence et l'instrument de distinction du polytechnicien au sein des élites sociales et politiques. Elle confère par ailleurs les normes du jugement rationnel à partir duquel se construit l'action technocratique et l'organisation de la bureaucratie d'État.

3 C'est l'intérêt de l'étude menée sur les principes de la formation pédagogique : il s'agit pour l'auteur de montrer comment la formation pédagogique et l'organisation des savoirs participent d'un « code scolaire » (système de règles et de normes régissant un univers social qui est transposé dans le système éducatif et le plan de formation). L'efficacité du système polytechnicien réside dans sa capacité à transformer des savoirs produits dans le cadre d'une activité savante en savoirs susceptibles de fournir à la technocratie des schèmes de pensée adaptés à leur mode de fonctionnement bureaucratique. De ce fait, l'École et son personnel jouent un rôle essentiel (pendant toute la première moitié du siècle) dans l'élaboration de la culture de gestion bureaucratique fondée sur la rationalité scientifique et mathématique. À travers cette analyse, l'auteur fait apparaitre les outils à partir desquels s'élaborent les principes cognitifs de l'action technocratique : la mise en place de la technocratie s'appuie sur une représentation particulière de la raison fondée sur l'application des sciences exactes et particulièrement des sciences mathématiques. Ce modèle constitue les fondements mêmes de la vision technocratique de l'action publique. Ainsi, l'École polytechnique s'impose comme le laboratoire de la «raison technocratique » qui se construit à travers la transformation de la science des savants en science des ingénieurs d'État. C'est à travers la mise en place de cet outil de l'action que se constituent également les modalités de la légitimité des polytechniciens. L'École convertit en quelque sorte le capital scientifique accumulé en un système d'outils nécessaires à l'action publique. L'auteur s'interroge sur les procédures de ce savoir et montre comment les mathématiques servent de support à un comportement spécifique qui se construit à travers le cursus de l'École : selon lui, les différentes étapes du cursus honorum obéissent à un ensemble de règles traduisant sur le plan didactique l'ensemble des règles d'un univers social dont il assure ainsi la reproduction. Avec la militarisation de l'École qui ne cessera de se renforcer, la place accordée aux mathématiques constitue ainsi le fondement essentiel de la transformation de l'École en l'espace de formalisation de l'univers technocratique. En un mot, l'École n'est pas seulement un espace de construction et de diffusion des connaissances techniques, mais plus encore garantit les fondements d'un univers social dont elle assure la conservation. Le parcours de formation contribue à construire une identité sociale et professionnelle qui s'apparente à la formation d'un ethos élitiste. C'est également comme instrument de la distinction entre «ingénieur civil » et «technocrate », une distinction spécifiquement française, que cette culture mathématique joue un rôle essentiel.

4 Contrairement à une formation pratique fondée sur l'imitation, la formation technique dispensée par Polytechnique est avant tout technocratique et abstraite, fondée sur l'analogie du calcul, particulièrement bien adaptée à la gestion de l'univers bureaucratique, mais de peu d'utilité dans l'univers technique. En subordonnant les savoirs pratiques aux savoirs théoriques (une des raisons de la critique traditionnelle portée contre les «technocrates»), il s'agit de construire l'autonomisation progressive 
de la figure sociale de l'ingénieur d'État. À l'idéal révolutionnaire, largement hérité de l'Ancien Régime, de former des ingénieurs-artistes succède un nouveau partage des compétences au sein de l'espace des techniques, Polytechnique devenant le lieu de formation des ingénieurs-bureaucrates et, en cela, une véritable succursale de l'administration d'État. Sur ce point, les années 1830 constituent un véritable tournant dans l'histoire de l'École. La militarisation commencée sous l'Empire se renforce puisque l'École est désormais placée sous l'autorité du Ministère de la Guerre. Cette militarisation se double d'une véritable spécialisation de la fonction assignée à l'École puisqu'elle fait complètement disparaître la mission d'intérêt général affirmée aux origines et maintenue par la réforme de 1816 : désormais, Polytechnique s'impose comme une succursale des « armes savantes ». Cette réforme menée par François Arago est lourde de conséquences quant à la position assignée à l'institution dans l'espace social. Face à l'émergence, à partir des années 1820 , de la figure de l'ingénieur « civil », de l'industriel et de l'entrepreneur qui, sur le modèle anglais, tendent à s'émanciper de la tutelle technocratique (et à battre en brèche le pouvoir symbolique des ingénieurs d'État) - émergence qui s'institutionnalise à travers la naissance de l'École centrale des arts et manufactures -, on assiste à un bouleversement radical des contours du champ « technique » qui n'est pas due à de nouvelles inventions, mais à une décomposition de la culture des arts et des sciences.

5 La militarisation de l'École doublée de la spécialisation des enseignements vers les mathématiques, s'inscrivent dans une stratégie de distinction qui permet à Polytechnique de se distinguer de la sphère civile et de conserver son lien étroit avec la technocratie. Face à la concurrence des ingénieurs civils et des entrepreneurs qui menacent l'existence même du corps polytechnicien, la réforme de 1830 élève une «barrière infranchissable » entre les deux univers (p. 145), barrière qui ne cessera de devenir de plus en plus étanche. En se renfermant vers les "armes", l'École se distingue ainsi des sciences civiles, mais conserve sa position de cadre technocratique. Le Second Empire marque l'élévation collective du corps des polytechniciens au sein de la haute fonction publique. La formation disciplinaire fondée sur un ordre des savoirs particulier donnant une large part aux savoirs théoriques (desquels doivent dépendre les savoirs pratiques) sont surtout là pour poser les bases de la distinction sociale du polytechnicien, plus que pour lui donner les clés d'un savoir pratique, susceptible d'être utilisé «sur le terrain ». La valorisation de la science mathématique (qui subit de nombreux infléchissements comme le souligne l'auteur) sert ainsi de fondement à la construction d'une posture et d'une distinction face aux ingénieurs civils et aux entrepreneurs. La normalisation progressive des savoirs entraîne en effet une intellectualisation des savoirs (qui laisse de côté -et contrairement à l'idéal de Mongeles observations de terrain ou la géométrie descriptive qui devaient réunir le savant et l'artiste). Les technocrates se distinguent ainsi des hommes de l'art par leur savoir et leur culture et peuvent revendiquer une autorité sociale, voire politique, fondée sur les méthodes, les pratiques et les outils scientifiques acquis pendant leur formation qui leur permettent de montrer des aptitudes particulières à la gestion et à l'administration (la technocratie "voit haut et loin »). La réforme de 1850 marque une nouvelle étape dans cet infléchissement: progressivement l'École cesse d'être un laboratoire scientifique pour s'imposer comme un espace de production de la «noblesse d'État ». Cette évolution est précisée dans la troisième partie consacrée à l'étude sociologique des élèves passés par l'École entre 1794 et 1870. L'entrée à l'École peut être considérée comme un véritable investissement (intellectuel et financier) qui 
suppose une stratégie individuelle, familiale et sociale (on trouvera des passages très intéressants sur les stratégies mises en œuvre pour s'ouvrir les portes de l'École) qui exclut de facto les catégories sociales les plus modestes, B. Belhoste s'écartant de certaines études qui affirmaient une certaine démocratisation du recrutement. L'étude souligne la sur-représentation des catégories «des capacités» (fonctionnaires liés à l'État) et des familles à fort capital culturel ainsi que la sous-représentation de la noblesse (ce qui constitue une rupture par rapport au recrutement des armes savantes pendant l'Ancien Régime).

6 L'auteur accorde une large place à l'étude du système de représentations à partir duquel se dessinent les contours de l'identité du groupe des Polytechniciens. Jusqu'en 1860 , la construction de la figure du «technocrate » est inséparable d'un idéal, d'une «mission» sociale et politique. La «méritocratie», fondement de l'idéologie polytechnicienne, valorise un "élitisme de l'intelligence » formalisée en large partie par les théories de Saint-Simon ou de Comte (que l'auteur présente comme des «intellectuels organiques » de l'École). On retrouve ici (après les Idéologues sous le Directoire) l'idéal d'une « République des savants » qui viendrait s'opposer à celui d'une "République des propriétaires ". La valorisation de ce système de valeurs et de représentations, particulièrement sous la Restauration, justifie ainsi la mission individuelle et collective que les Polytechniciens s'assignent dans la société : une mission d'encadrement et d'éducation de la Nation qui viendrait concurrencer le pouvoir traditionnel des notables fondé sur la naissance et la propriété. L'idéal de service de l'État n'est donc pas incompatible avec celui de " guide " politique dont se réclament les Polytechniciens qui cherchent à mettre en pratique les outils technocratiques dans d'autres espaces que celui de la bureaucratie (la philanthropie ou l'éducation populaire), traçant ainsi les contours d'un véritable "pouvoir spirituel ", sorte de sacerdoce scientifique. Dans la continuité de cette mission, un idéal républicain imprègne l'École comme le souligne la participation particulièrement active des Polytechniciens aux révolutions de 1830 et 1848, participation qui donnent d'ailleurs naissance à un véritable mythe du Polytechnicien, figure sociale de l'intelligence «mise au service» du peuple (homme d'ordre, mais proche du peuple). La reprise en main de l'École par les autorités militaires, mais également l'évolution des tensions entre l'idéal révolutionnaire et le service de l'État expliquent que cet idéal s'efface à partir de 1851 pour disparaître complètement par la suite, les Polytechniciens se retrouvant aux côtés des Versaillais lors de l'écrasement de la Commune de Paris en 1871. Pour le Polytechniciens, il s'agit désormais de renforcer leur position dans les sphères de pouvoir et de compenser la perte de domination sur le champ technique par un renforcement de l'influence sur la sphère dirigeante (alliance avec les hommes d'affaires et les élites politiques). Alors qu'ils auraient pu se fondre dans une profession d'ingénieurs associée à l'industrie, les Polytechniciens (le terme s'impose dans les années 1860) décident de renforcer leur vocation régalienne et intègrent la «noblesse d'État » sous le Second Empire dont ils partagent progressivement les origines, les intérêts et les convictions. Pour l'auteur, les années 1860-1870 marquent une évolution importante : l'École se « fige » (elle apparaît alors plus un « conservatoire des sciences » qu'un espace de recherche) et cesse d'être un centre d'innovation pour devenir un espace de conservatisme politique et social tout en étendant son emprise sur la gestion des appareils industriels, une constatation qui semble justifier le pessimisme affiché par l'auteur dans son introduction quant à l'avenir d'une institution qui n'apparaît plus aujourd'hui que comme un instrument de domination sociale et politique. 
7 On l'aura compris, cet ouvrage, à la croisée de l'histoire des sciences et de l'histoire des institutions d'État, constitue déjà une contribution importante à l'analyse des logiques de construction des groupes sociaux pendant le XIXe siècle. 